\title{
Grey Pages Rapid publication of conference reports
}

\section{JOHN FOX}

Each year many small and medium sized workshops and conferences are organised on emerging topics in and around AI and knowledge engineering. Such meetings are often stimulating, and sometimes seminal, but attendance is limited and the proceedings only make it into the "grey literature", if they are published at all. This issue contains two examples of Grey Pages, a new kind of article that provides a way of informing readers about developments that are important, or are expected to become so, for the applied AI or Knowledge Engineering communities.

Unlike many conference reports, which frequently only record conference highlights or give a blowby-blow summary of contributions, we are looking for succinct "mini-papers"; thoughtful pieces that provide the background to and main themes of the meeting. The aim should be to alert the research and development communities to areas that should be watched. Features in Grey Pages will be fully citable.

\section{A Model for Grey Pages}

The general tone and style of Grey Pages can be quite informal, aiming to persuade readers why a new area is likely to be important rather than presenting technical detail. However, the readership of the Review is very broad, and emphasis should be placed on presenting adequate background to the topic and providing sufficient motivation. A suggested, though not mandatory, structure is:

Title

Meeting details

(No abstract)

1. Introduction $(=<500$ words)

2. Background and motivation ( $=<750$ words)

3. Principle themes of the meeting $(=<750$ words)

4. Assessment and projections ( $=<500$ words)

5. Conclusions $(=<250$ words)

6. Details of availability of proceedings, references, and further reading.

Finally, if a topic has clear relevance to readers of the Review it can be considered for detailed treatment in a feature article. The editor is happy to receive advice on whether to commission an extended survey or special issue, and on possible contributors.

\section{Submission and review}

Articles should be about 1000-3000 words. They should be submitted by email to the editor (internet address jf@acl.icnet.uk). Please send submissions in plain ascii text. If there are special formatting requirements these can be illustrated by supplying a LateX version in a separate email, or by fax to the editor on +44-71-269-3186. To expedite publication the decision about acceptance may be taken solely by the editor. If an article is accepted, it will appear in the next available issue of the Review. 